avenues need to be sought to ensure prompt treatment and prevent relapse.

A CTO suite designed for recall could be the solution for the future.

\section{Overdose admissions to a district general hospital intensive care unit}

Aoife Nechowska*, Theophilus Samuels and Sameer Ranjan

Surrey and Sussex Healthcare NHS Trust

${ }^{\star}$ Corresponding author.

doi: 10.1192/bjo.2021.285

Aims. This audit aimed to analyse the patient population coming into East Surrey Hospital's Intensive Care Unit from 1993 to 2019. Background. The Office for National Statistics (ONS) published a report in August 2019 on ages most likely to die by suicide and drug poisoning. Their data showed that Generation $\mathrm{X}$ were dying by this method in greater numbers than other age groups. This is in contrast with data from 1990s for England and Wales which showed people in their 20s were most likely to die by suicide or poisoning. This audit set out to look at admission data from an intensive care unit (ICU) in a district general hospital in Surrey over a similar period of time.

Method. Patient records from 1993 to September 2019 were accessed using the WardWatcher database. To access the maxim number of admissions qualifying under the aims, the database was accessed by searching under "admission comments" for: overdose, self-harm, poison, suicide. These reports were downloaded and the lists were checked against each other to delete duplicates. This gave a total of 331 patients. The data were analysed by year, according to age, gender, season, psychiatric diagnoses and previous overdose attempts. Their outcomes were checked against recorded deaths. There was not enough information to investigate method of overdose.

Result. A total of 331 patient records were accessed. The youngest patient was 15 years old, the oldest was 84 years old. The age dataset was non-parametrically distributed with the median age of 43 years (IQR 33-51 years). The age distributions for each year appeared symmetrical but total numbers for each year were small. The population was split as 191 female (58\%) and 141 male (42\%). 16 patients died on the ICU on admission with an overdose, $5 \%$ of total numbers, of which $19 \%$ had a previous overdose attempts and $44 \%$ had a psychiatric diagnosis. The youngest death was 22 and the oldest was 81 years old. The average age was 47 years, with the spread consistent in the 2000s and 2010s.

Conclusion. The results from East Surrey Hospital's ICU do not reflect the analysis from the ONS. The mean age for each year has remained similar. Numbers for the audit were small and admission criteria to the ICU prescribe that the patient be critically unwell and may not be indicative of the total admissions to a district general hospital.

\section{Neuroimaging in an older adult inpatient psychiatric unit}

Aoife Nechowska

Surrey and Borders Partnership NHS Foundation Trust

doi: 10.1192/bjo.2021.286

Aims. This audit aimed to analyse the use of neuroimaging and its effect on treatment in an older adult inpatient psychiatry unit over the period of one year.
Background. Brain imaging can be used as a diagnostic tool in psychiatry. According to NICE guidelines, structural imaging, such as magnetic resonance imaging (MRI) or computed topography (CT), can be used in the workup for dementia diagnosis in order to exclude non-dementia pathology and identify dementia subtype. This is important in the geriatric population where evidence of small vessel disease has an impact on treatment options and management of polypharmacy.

Method. A list of patients from the past year (January to December 2019) was accessed. Patient records were then analysed to see if neuroimaging had been accessed during their admission at The Meadows Hospital, Surrey and Borders Partnership. This included imaging from prior to admission. Analysis was divided into type of imaging, comments and impact on diagnosis.

Result. Overall numbers for the audit were small. A total of 74 patients were admitted onto the unit, of which 3 were readmissions. There was missing information for 8 patients, giving a total of 63 patients. CT scans were accessed for 35 patients ( $56 \%$ of total); 3 of these were done during the admission. MRI scans were completed for 21 patients (33\%), with one requested during admission. A total of 9 patients (14\%) had both CT and MRI scans. Neuroimaging results led to a change in diagnosis for 6 patients (10\%). In all cases this reflected the finding of small vessel disease and a change of diagnosis to either vascular dementia or mixed dementia.

Diagnoses were also analysed. The Meadows Hospital has 2 dementia wards (male and female) and 1 functional ward (for females). A total of 36 patients (57\%) were diagnosed with dementia, of which the biggest groups were: Alzheimer's dementia (13 patients, $36 \%$ ) and Vascular dementia (11 patients, $31 \%$ ). Conclusion. The majority of the patients were admitted with established diagnoses and so only a small number had changes made following review of imaging. Good imaging results and reports help to differentiate types of dementia. Although neuroimaging is not gold standard in diagnosing dementia syndromes, it is now an important aspect in the diagnostic pathway. Getting the diagnosis correct will help with treating individuals appropriately and avoid unnecessary prescribing.

\section{Implementation of smoking cessation policy at the} antelope house in Southern Health NHS Foudation Trust in Southampton

Sunday Neru* and Edet Ededet

Antelope House, Southern Health NHS Foundation Trust, Brintons Terrace, Southampton

${ }^{\star}$ Corresponding author.

doi: 10.1192/bjo.2021.287

Aims. To ensure that health care practitioners at Antelope house, Southern Health NHS Foundation Trust in Southampton are providing service users information, advice and stop smoking support in line with smoke free policies in the trust.

Background. Smoking is the single largest preventable cause of ill health and premature death in England. Cigarette smoking causes a wide range of diseases and medical conditions like cancers, heart diseases and stroke.

The prevalence of stroke is extremely high among people with mental health problems especially those admitted to hospital.

Stopping smoking reduces the risk of developing preventable diseases and premture death.

These are the background behind this audit. 